\title{
Tecnologias e métodos que auxiliam na comunicação de surdocegos: uma revisão bibliográfica
}

\author{
Patricia F. Amorim ${ }^{1,2}$, Simone B. Leal Ferreira ${ }^{2}$, Priscyla G. F. Barbosa ${ }^{2}$, \\ Humberto S. Pacheco ${ }^{2}$, Aline da S. Alves ${ }^{2}$ \\ ${ }^{1}$ ISERJ - Instituto Superior de Educação do Rio de Janeiro \\ Rio de Janeiro - RJ - Brasil \\ ${ }^{2}$ UNIRIO - Departamento de Informática Aplicada \\ Rio de Janeiro - RJ - Brasil \\ \{patrícia.amorim, simone, priscyla.ferreira\}@uniriotec.br, \\ \{humbertocin, aline.ihc\}@gmail.com
}

\begin{abstract}
This article presents the results of a first analysis of a bibliographical research that explores the approaches of the works already carried out in the theme of multiple deficiencies focused on people with deafblindness. In order to identify the technologies, languages and device types used in deafblind communication, somehow help their education. Preliminary results shows that papers used as main language to establish communication, braile and its derivations: bodybraile, fingerbraile, and a predominance of resources for bidirectional communication in the interaction of deafblind individuals. Few papers have explored methods or resources for learning.
\end{abstract}

Resumo. Este artigo descreve a primeira análise de uma pesquisa bibliográfica que explora as abordagens dos trabalhos realizados na temática de deficiencias múltiplas focado em pessoas com surdocegueira. Com a finalidade de identificar as tecnologias, as linguagens e os tipos de dispositivos utilizados na comunicação dos surdocegos e, que de alguma forma, auxiliem a sua educação. Como resultado inicial, identificou-se como principal linguagem para estabelecer a comunicação, o braile e suas derivações: bodybraile, fingerbraile, e uma predominância de recursos para a comunicação bidirecional na interação de indivíduos surdocegos. Foram poucos trabalhos que exploraram métodos ou recursos para aprendizagem.

\section{Introdução}

A área de acessibilidade vem crescendo cada vez mais no Brasil através do avanço na promoção dos direitos das pessoas com deficiência por meio das políticas públicas (Ferreira, 2012). Houve também um aumento no número de trabalhos publicados em eventos como uma preocupação crescente de grupos de pesquisa em abordar este assunto (Granato, 2016). No ano de 2006, a Sociedade Brasileira de Computação (SBC) propôs definir os Grandes Desafios para as Pesquisas em Ciência da Computação no Brasil pelos próximos 10 anos e o quarto grande desafio foi "Acesso participativo e universal do cidadão brasileiro ao conhecimento".

A comunidade da área de Interação Humano-Computador (IHC), também pensando nos desafios de pesquisas para os próximos anos, lançou os "Grandes Desafios da Pesquisa em Interação Humano-Computador no Brasil” (GrandIHC-BR) 
indicando o tema de Acessibilidade e Inclusão Digital (Granatto, 2016). Em 2017, a comunidade IHC apresentou como segundo grande desafio o tema "Acessibilidade e Inclusão Digital: Utopia ou Grande Desafio?” (Ferreira et al., 2017), uma reflexão baseada nos últimos cinco anos, que descreveu alguns resultados já encontrados e identificou um conjunto de questões a serem tratadas pela comunidade brasileira de IHC. Dentre este conjunto de questões (CQ), o segundo CQ trata do tema de melhorias e/ou adaptações a serem feitas nas tecnologias assistivas existentes para proporcionar maior autonomia ao deficiente brasileiro, analisando a construção e avaliação de interfaces acessíveis a indivíduos com múltiplas deficiências. Também recomendou uma agenda para as futuras pesquisas nos próximos cinco anos, tendo como itens: o fomento ao desenvolvimento de interfaces, que considerem os variados contextos e a diversidade cultural brasileira e promover recomendações de IHC especializadas em deficiências cognitivas, neuronais e de aprendizagem (Ferreira et al., 2017).

Considerando o universo de Sistemas de Informação, a comunidade apresentou recentemente os Grandes Desafios da Pesquisa em Sistemas de Informação 2016 a 2026(GranDSI-BR), indicando como primeiro grande tema, Sistemas de Sistemas, que tem como terceiro item, Desafios para Sistemas de Informação na Implementação do Conceito de Cidades Inteligentes no Brasil, como Interação Humano-Computador, na construção de interfaces e estudo de sistemas mais amigáveis e acessíveis aos usuários dos sistemas das Cidades Inteligentes, onde percebe-se a preocupação em produzir conhecimento que permita acesso universal a todos nas futuras Cidades Inteligentes (Araujo, et al., 2017). O que mostra uma preocupação geral por pesquisas que abordem os temas de acessibilidade.

O trabalho de Granatto (Granatto et al., 2016) mapeou a evolução das pesquisas relacionadas a área de acessibilidade apresentadas no Simpósio Brasileiro sobre Fatores Humanos em Sistemas Computacionais (IHC) de 1998 a 2015. O trabalho procurou identificar quantos trabalhos publicados no IHC possuem como foco temas relacionados à Acessibilidade Digital. Segundo o levantamento deste trabalho, os principais temas abordados até esta edição foram: deficiências gerais, deficiência visual, deficiência auditiva, motora e terceira idade. Não foram identificados trabalhos que abordassem as deficiências múltiplas como é o caso da surdocegueira.

Surdocegueira é uma deficiência reconhecida como única condição incapacitadora que apresenta características de perdas auditiva e visual, levando quem a possui a ter formas específicas de comunicação para ter acesso a lazer, educação, trabalho e vida social (Cader-Nascimento, 2010). O indivíduo é privado então de receber informações de ordem simbólica, temporal e direcional, assim a compensação sensorial do mesmo caracteriza-se por meio de outros sentidos perceptivos como tato e olfato (Bertola, 2014).

Esse quadro específico de deficiência múltipla gera necessidades especiais de comunicação, causando em alguns casos, extrema dificuldade em acessar informações e compreender o mundo externo, o que resulta num isolamento.

O presente trabalho pretende analisar, especificamente, artigos internacionais catalogados no eixo temático "Surdocegueira" e "Ciência da Computação" para investigar e identificar as barreiras enfrentadas por usuários surdocegos na compreensão de conteúdos. O problema de pesquisa é a dificuldade dos surdocegos compreenderem informações textuais ou gráficas. Esses usuários possuem uma forma de comunicação 
diferente dos surdos e dos cegos: eles precisam de um contato físico para estabelecer a comunicação, sempre contando com a presença de um intérprete. Atualmente há muita dependência dos intérpretes para a aquisição de conteúdos no contexto da surdocegueira.

Após a identificação das principais barreiras que dificultam a compreensão e a comunicação do conteúdo por esse público, pretende-se sugerir recomendações que possam contribuir para um melhor entendimento para o desenvolvimento de artefatos de TI para surdocegos.

A tecnologia pode ser uma grande aliada para auxiliar na comunicação de pessoas com surdocegueira. Em diversos trabalhos levantados, o uso de motores vibradores em luvas, roupas e smartphones estimulam o tato para transmitir informações de alguma língua, processo de leitura/escrita (como o braile) ou codificação (como o morse). Nesse contexto de soluções tecnológicas para pessoas com surdocegueira, o presente trabalho tem por objetivo realizar uma pesquisa exploratória através de um levantamento bibliográfico na área de Sistemas de Computação, para identificar as tecnologias, as linguagens e os tipos de dispositivos (artefatos de TI) utilizados na comunicação dos surdocegos entre si ou com pessoas sem deficiência e, que de alguma forma, possam servir de base para a proposta de um método que a auxilie o processo ensino-aprendizagem dos indivíduos surdocegos.

Os resultados apontam para a predominância de trabalhos que criaram recursos para a comunicação bidirecional na interação de indivíduos surdocegos com pessoas sem deficiência numa troca de envio e recebimento de mensagens. No estabelecimento da comunicação, os trabalhos utilizaram como principal linguagem, o braile e suas derivações: bodybraile, fingerbraile. Muitos trabalhos considerados na pesquisa não exploraram métodos ou recursos para aprendizagem. Desta forma, não é possível afirmar que efetivamente melhorem o desenvolvimento do surdocego num processo de ensino-aprendizagem.

O artigo está estruturado da seguinte forma: a seção dois apresenta a fundamentação teórica sobre surdocegueira e comunicação; a seção três apresenta a metodologia adotada para a pesquisa bibliográfica; a seção quatro apresenta os resultados compilados da análise inicial dos dados obtidos; a seção cinco apresenta as tecnologias e métodos identificados para a comunicação de pessoas surdocegas; e, finalmente a seção seis apresenta as conclusões deste trabalho e propostas de trabalhos futuros.

\section{Fundamentação Teórica}

\subsection{Surdocegueira}

O termo adotado no estudo "surdocegueira", surgiu em 1995, porém antes do estabelecimento deste termo, segundo Frederico (Frederico, 2006) existiram vários outros como: Dificuldade de Aprendizagem Profunda e Múltipla (DAPM), Múltipla Deficiência Severa, Cego com Deficiência Adicional, Múltipla Privação Sensorial (MPS) e dupla Deficiência Sensorial.

O conceito para surdocegueira, segundo a publicação do Grupo Brasil, instituição civil de caráter social, é: "uma deficiência singular que apresenta perdas auditivas e visuais concomitantemente em diferentes graus. Levando a pessoa surdocega 
a desenvolver diferentes formas de comunicação para entender e interagir com as pessoas e o meio ambiente, proporcionando-lhes o acesso a informações uma vida social com qualidade, orientação mobilidade, educação trabalho." (GRUPO BRASIL, 2003).

Uma pessoa surdocega, tem a combinação de déficit simultâneo da visão e da audição, privando-a de receber informações de ordem simbólica, temporal e direcional, assim a compensação sensorial e interação desta pessoa com o meio a sua volta se dará pelos sentidos táteis e olfativos.

A surdocegueira é classificada de diversas formas. Segundo o ponto de vista sensorial ela é classificada em quatro níveis: 1 - indivíduos surdos profundos e cegos; 2 indivíduos surdos e que têm pouca visão; 3 - indivíduos com baixa audição e que são cegos; 4 - indivíduos com alguma visão e audição. Quanto ao período do surgimento desta condição: pré-linguística (congênita) antes da aquisição da linguagem, seja oral ou gestual; ou pós-linguística (adquirida) depois da aquisição da linguagem McInnes (McInnes, 1999).

As principais causas da surdocegueira variam de acordo com a época de aquisição. Surdocegueira congênita está relacionada com infecções transmitidas por rubéola, meningite, citomegalovirus, toxoplasmose, causas genéticas ou síndromes como síndrome de Goldenhar, síndrome de CHARGE ou problemas pré-natais como a prematuridade, baixo peso ou trauma. Nos casos da surdocegueira adquirida as causas podem ser acidentais, relacionadas ao envelhecimento ou síndrome genética como a Síndrome de Usher (Maia, 2012 appud Aitken, 2000).

Para possibilitar uma melhor compreensão sobre a pessoa com surdocegueira e pesquisar tecnologias desenvolvidas com o objetivo de ajudar na comunicação desses indivíduos e as pessoas que atuam no seu dia a dia num processo de ensinoaprendizagem, é necessário considerar alguns conceitos sobre comunicação e quais recursos utilizados para efetivá-la.

\subsection{Comunicação}

A comunicação é a interação entre humanos através de símbolos e sistemas de mensagens (Blumer, 1980). A criança surdocega, que ainda não está inserida num contexto de comunicação usando algum tipo de recurso, utiliza normalmente formas de comunicação não verbais tais como sorrisos, movimentos, mudanças de posição que são compreendidos por adultos familiarizados com esses gestos (Nascimento, 2006). Os recursos de comunicação usados por crianças surdocegas são: sistemas alfabéticos tais como os datilológicos, letras maiúsculas, tablitas, braile; e os sistemas não alfabéticos tais como a LIBRAS adaptada, leitura labial, Tadoma, movimentos corporais, sinais no corpo, símbolos, sistemas suplementares de comunicação como levantar a cabeça, desenhos e outros" (Nascimento, 2006).

Para compreender como as pessoas se comunicam é necessário considerar os sentidos que são os meios através dos quais os seres vivos percebem e reconhecem o mundo físico a sua volta e processam as informações no seu cérebro (Garcia, 2008).

O sistema sensorial é formado por células ou terminações nervosas encontradas nos órgãos dos sentidos do corpo. Através deles os seres humanos conhecem a existência dos objetos que os cercam. Estes objetos são identificados ao ver sua forma e 
cor, ouvi-los, tocá-los e muitas vezes degustá-los. Todas as informações captadas pelos órgãos sensoriais se convertem em sinais eletroquímicos, que são enviados ao cérebro para serem processados e transformados em estímulos ou armazenados na memória. As informações das sensações (estímulos) possuem quatro parâmetros importantes: a modalidade, a intensidade, a duração e a localização. Além dos sentidos fundamentais, o ser humano possui o sistema somático, sistema responsável pelas percepções das sensações da pele e da superfície do corpo, como o tato, a dor, a pressão, a posição e o movimento. O sistema somático é fundamental na comunicação do surdocego e na exploração do mundo a sua volta, já que o seu sistema auditivo e visual estão comprometidos (Garcia, 2008).

Assim, para ocorrer a comunicação com um surdocego é necessário estar perto dele, sempre tocá-lo para apoiar e aumentar sua compreensão, e observar todas as maneiras com as quais ele está se comunicando e usar adaptações adequadas à cada caso.

\section{Método de Pesquisa}

Foi adotada a metodologia de levantamento bibliográfico sugerido por Vosgerau e Romanowski (Vosgerau e Romanowski, 2014). A pesquisa teve as seguintes etapas: (a) definição das estratégias de buscas; (b) seleção de estudos relevantes; (c) análise e avaliação dos estudos; (d) processo de seleção dos estudos primários e (e) extração e síntese das informações relevantes.

\subsection{Etapas da Pesquisa}

a) Definição da estratégia de busca

Foi utilizada a base de busca Scopus (http://www.scopus.com) para identificação das pesquisas relevantes que tratam o estado da arte dos métodos e ferramentas para apoio à surdocegos. Foi definido o período de 2000 a 2016 para levantar as ocorrências nos últimos 16 anos. Os conceitos e seus termos sinônimos utilizados na busca foram: deafblindness, deaf-blind e usher syndrome.

b) Seleção de estudos relevantes

A pesquisa dos trabalhos na base Scopus foi realizada utilizando as strings de busca (Tabela 1) dos conceitos na língua inglesa sempre para os mesmos metadados: títulos, resumos e palavras-chaves. Também foi definido que somente trabalhos da área de Ciência da Computação seriam analisados visto que esse tema é bastante recorrente em outras áreas (Figura 1) como: Medicina, Ciências Sociais, Matemática, Engenharia, etc.

Tabela 1. Strings de busca e resultados. Fonte: Elaborado pelos autores

\begin{tabular}{|c|c|}
\hline String de busca & Resultados \\
\hline $\begin{array}{c}\text { TITLE-ABS-KEY ("deafblindness" OR "deaf-blind" OR } \\
\text { "usher syndrome") AND PUBYEAR > 1999 AND } \\
\text { (LIMIT-TO (SUBJAREA, "COMP" ) }\end{array}$ & 73 \\
\hline
\end{tabular}




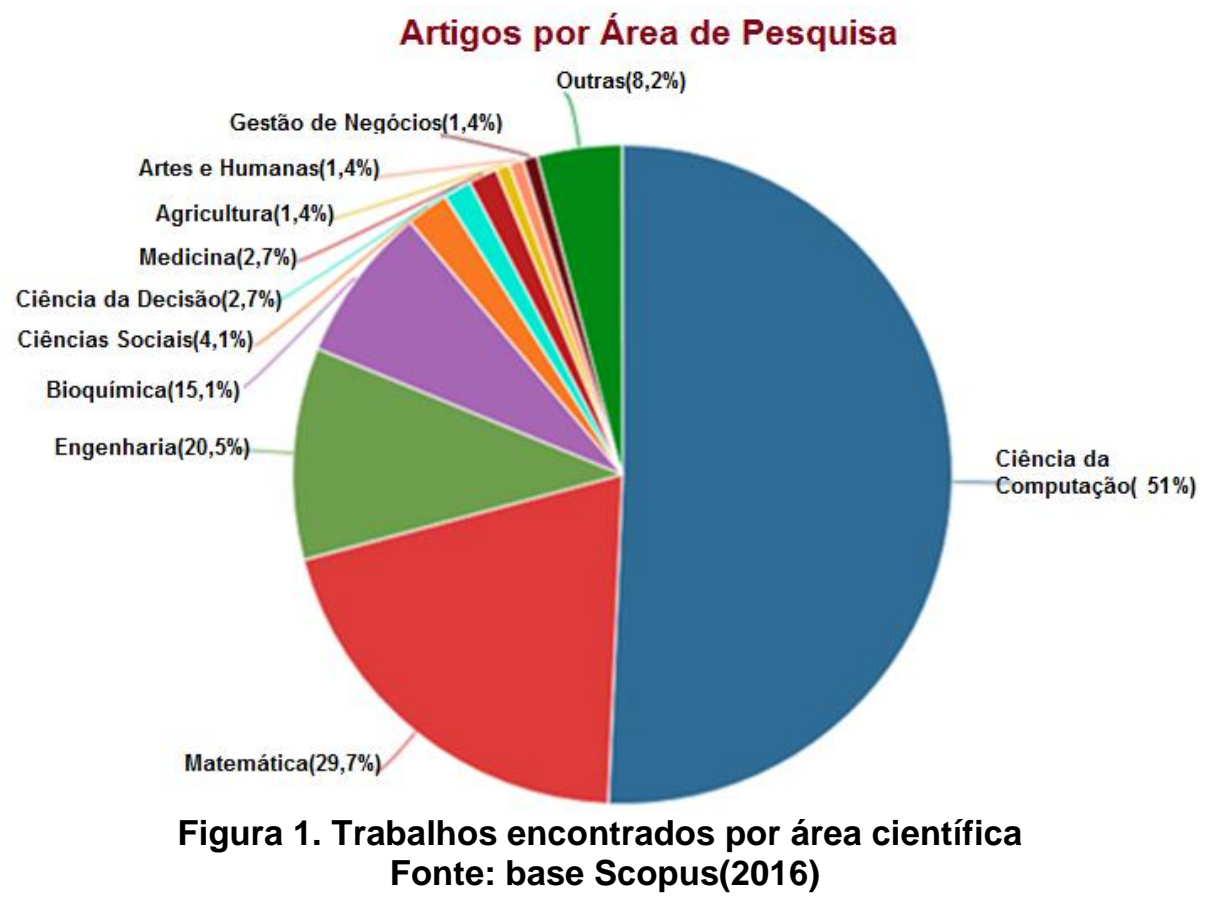

Ao fazer uma análise por país de publicação (figura 2) apenas dois trabalhos identificados na base Scopus eram do Brasil. O que poderia indicar uma necessidade de estudos na área de Computação para a realidade brasileira dos usuários surdocegos.

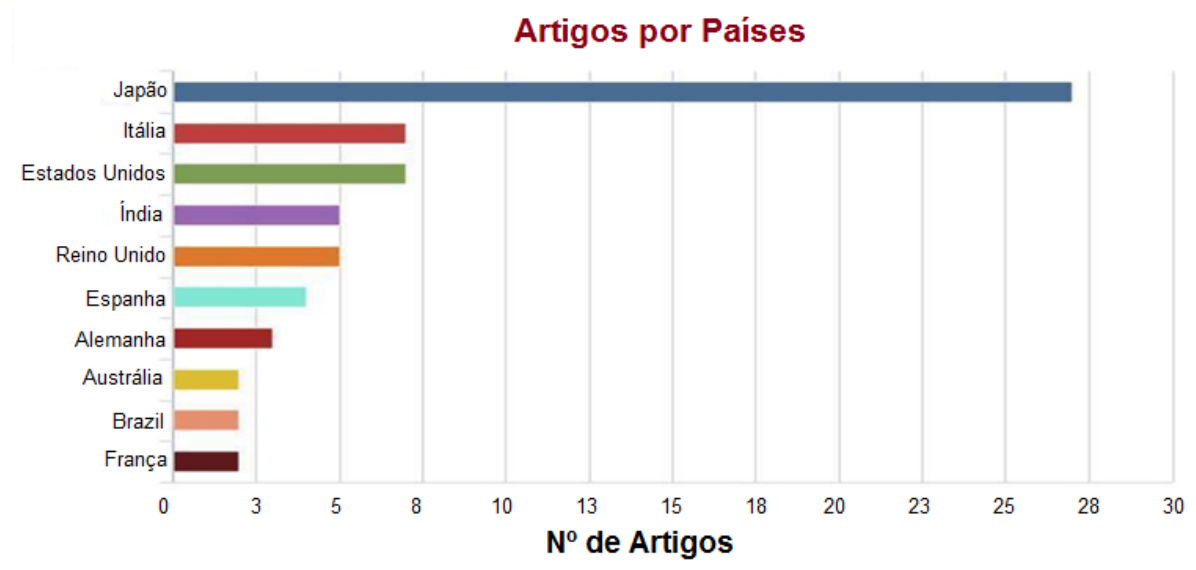

Figura 2. Trabalhos por país de publicação

Fonte: base Scopus(2016)

c) Análise e avaliação dos Estudos

Foram analisados os títulos, resumos e palavras-chave de cada artigo a fím de decidir se os estudos encontrados na pesquisa deveriam ou não ser incluídos na avaliação seguindo os seguintes critérios de inclusão e exclusão para a próxima etapa de análise dos estudos.

Critério de Inclusão: Trabalhos que apresentam artefatos que possam auxiliar na educação ou comunicação de pessoas com surdocegueira.

Critério de Exclusão: Trabalhos que não tratam da surdocegueira entendida como uma deficiência múltipla, mas sim de limitações na visão ou na audição ou que tratem exclusivamente de uma das duas deficiências: surdez ou cegueira. 
d) Processo de seleção dos estudos primários

O processo de seleção dos estudos primários do levantamento bibliográfico foi baseado nas orientações sugeridas por Vosgerau e Romanowski (Vosgerau e Romanowski, 2014). Para possibilitar a documentação desse processo, facilitar a extração e sumarização dos dados, foi elaborado o "Formulário de Seleção e Condução de Estudos", contendo os seguintes campos: nome do artigo, lista de autores, ano de publicação, veículo de publicação do artigo, tipo de publicação, Nível Qualis Capes, tecnologias envolvidas e como as tecnologias propostas ajudam na comunicação de pessoas com surdocegueira. A seguir são apresentados os passos do processo de seleção dos estudos primários:

1. Realizar o processo de busca dos artigos na base selecionada;

2. Os artigos encontrados são documentados na lista de artigos presente no Formulário de Seleção e Condução de Estudos;

3. Os artigos documentados são verificados pelos critérios de inclusão e exclusão estabelecidos, essa verificação é executada mediante a leitura do título, resumo e palavras chaves do artigo;

4. Os artigos incluídos e excluídos são documentados no Formulário de Seleção e Condução de Estudos, juntamente com a justificativa de sua inclusão ou exclusão;

5. Os artigos incluídos são avaliados mediante a leitura do artigo completo.

e) Extração e síntese das informações relevantes

O levantamento bibliográfico foi executado entre o final de 2016 e início de 2017 , durante um período de quatro meses. Ao todo foram analisados 73 artigos dos quais 49 foram selecionados e tiveram os seus dados analisados, de acordo com as recomendações previstas no processo de seleção dos estudos primários e os critérios de inclusão e exclusão.

Aplicando-se o critério de exclusão descrito na etapa c, foram rejeitados 24 artigos. Esta rejeição foi realizada por meio de uma leitura criteriosa dos resumos e das introduções dos artigos. Aplicando-se o critério de inclusão, foram aceitos todos os demais 49 artigos porque esses apresentaram artefatos que podem auxiliar na comunicação de pessoas com surdocegueira e futuramente ser base para um trabalho com surdocegos aplicado a um processo de ensino-aprendizagem.

Para auxiliar na identificação e sistematização dos estudos que seriam incluídos na análise foram extraídos os seguintes fatores: título, autores, ano da publicação, país de origem, idioma, tipo de publicação (periódico ou conferência), instituição, evento de publicação, nível qualis Capes, método, objetivo, tecnologias. Bem como foram anexados os critérios de inclusão ou exclusão de cada trabalho para serem avaliados pelos pesquisadores.

Para classificar os dados extraídos dos trabalhos foi utilizado o formulário de extração de dados: formulário contendo campos para registro das análises e resultados do estudo, além de comentários adicionais do pesquisador que extraiu os dados. Esse formulário identificou os seguintes fatores: comunicação (unidirecional ou bidirecional); língua adotada (Braile, Sinais, Morse, Lorm); tecnologias ou recursos (vibrotátil, tátil, bluetooth, RFID, GPS, multimídias, outros); dispositivo (vestível, 
smartphone, smartwatch, robô, outros). Desta forma então foi possível avaliar e realizar as comparações apresentadas na próxima seção.

Após a análise dos 49 artigos, somente dois estavam dentro do escopo desta pesquisa, e três artigos estavam próximos, considerando que a proposta poderia gerar a apropriação de conhecimento. Os demais abordavam a análise e o registro de desenvolvimento de aplicativos e dispositivos que instrumentalizavam o desenvolvimento de uma comunicação básica entre as pessoas envolvidas nas pesquisas, que nem sempre eram surdocegos, mas que visaram descobrir e ampliar pesquisas na área da Surdocegueira. Observou-se somente um artigo de origem brasileira.

\subsection{Limitação}

Até o presente momento a principal limitação desta pesquisa é o baixo número de indivíduos surdocegos que aceitem essa caracterização e que sejam residentes no Rio de Janeiro.

\section{Resultados}

Durante a análise, percebeu-se que os trabalhos abordavam a comunicação receptiva, recebimento e compreensão de mensagens, e a comunicação expressiva, envio e expressão. Cerca de $75 \%$ dos trabalhos selecionados trataram da comunicação bidirecional quando era possível observar a interação de indivíduos surdocegos com pessoas sem deficiência numa troca de envio e recebimento de mensagens. No entanto cerca de $25 \%$ trataram da comunicação unidirecional focando apenas numa dessas etapas da comunicação, na emissão ou na recepção das mensagens pelos indivíduos surdocegos.

Para estabelecer a comunicação, os trabalhos utilizaram diversas línguas ou códigos como o braile, bodybraile, fingerbraile, língua de sinais, código Morse, Lorm (vocabulário mapeado nas mãos, alfabeto manual) com o propósito de auxiliar na leitura, escrita, tradução e aprendizado. O sistema Braile e suas derivações, bodybraile, fingerbraile, foram os mais abordados nos artigos, $57 \%$ do total dos artigos analisados. Para os outros códigos abordados obteve-se 18\% língua de sinais, 6\% código Morse, $6 \%$ alfabeto manual e $6 \%$ outras.

Quanto às tecnologias e os recursos estudados encontrou-se a utilização de $77,5 \%$ vibrotátil, $18 \%$ tátil, $10 \%$ Bluetooth, $6 \%$ GPS, $20 \%$ recursos web, $10 \%$ arduino, e $28,5 \%$ multimídias, enquadrados nesta categoria os dispositivos de saída e entrada de sons e imagens como câmeras, microfones ou fones. Essas tecnologias deram suporte aos dispositivos desenvolvidos ou aplicados nos estudo encontrados tais como dispositivos vestíveis como coletes ou luvas, smartphone, smartwatch, robôs ou hardwares específicos. A categorização por tipo de dispositivos utilizados nas pesquisas foram os seguintes: $22 \%$ dos artigos utilizaram dispositivos vestíveis, $31 \%$ smartphone, 4\% smartwatch, 10\% robôs. Em 37\% dos artigos considerados os pesquisadores desenvolveram um dispositivo específico para a pesquisa.

Dentre os 49 trabalhos selecionados, somente o (Vieira et al., 2016) era do Brasil. Esse artigo foi baseado no trabalho da equipe de iniciação científica da Universidade Centro Estácio Juiz de Fora que estuda e pesquisa as possibilidades de automação usando o Arduino, placa eletrônica única para prototipagem de hardware livre e baixo custo (Wikipédia, 2018). A proposta era criar um aplicativo para 
smartphone Android, que permitisse a comunicação entre indivíduos surdocegos e também entre surdocegos e pessoas sem deficiência. O protótipo do aplicativo apresentado no trabalho utilizava um módulo bluetooth full-duplex para a comunicação (envio e recepção de dados) entre smartphones, como também convertia texto em código Morse, que se transforma em vibrações para a pessoa surdocega. $O$ artigo informa que a pesquisa ainda estava em fase de testes com a instituição parceira e que para estudos futuros estavam incluídas implementações no projeto tais como: alterações na interface do aplicativo para melhorar a usabilidade do dispositivo Android; inclusão de sensor ultrassônico para detecção de objetos e obstáculos próximos aos surdocegos e para aqueles que ainda têm pouca visão ou audição, a inclusão de LED e BUZZER.

Ao identificar e analisar resultados de pesquisas que estudaram o processo ensino-aprendizagem e a comunicação de pessoas com surdocegueira, foram encontrados somente dois artigos. O primeiro é o de Zeza e Stavrou (Zeza e Stavrou, 2015), porém esse, não fez uso de dispositivo tecnológico. O trabalho era uma pesquisa qualitativa, exploratória e descritiva com o objetivo de desenvolver uma abordagem intervencionista de educação para um estudante surdocego com dificuldades rastreadas no esquema de consciência do corpo. Foram oferecidas ao aluno experiências multissensoriais e concretas para consciência das partes do corpo. Após a execução das atividades sensoriais, observou-se que o aluno ficou ciente de ter um corpo com um centro (linha média) e dois lados. O estudante conseguiu nomear as partes do corpo e identificar essas partes do corpo em outras pessoas.

O segundo artigo é o de Gorodnitsky et al. (Gorodnitsky et al., 2011). Este artigo descreveu o projeto "Dashpoint", um sistema que engloba hardware e software projetado para o e-learning de surdos, cegos e surdocegos. O hardware projetado foi um dispositivo háptico, que tem características do sistema sensorial tátil, para receber ou emitir um estímulo depende do contato direto com o objeto tocado (Sherman e Craig, 2003). O dispositivo apresentado neste trabalho trata do envio e recebimento de informação semelhante a um mouse, permitindo a transmissão dos símbolos do alfabeto Braile. No momento da publicação deste artigo, o projeto "Dashpoint" era um conjunto de serviços, incluindo um aplicativo para computador pessoal (PC) e um aplicativo para Windows Phone 7. Os serviços oferecidos eram chamados de gates e continham os seguintes itens: Edu.gate, Comuni.gate, Social.gate, info.gate. Suas funções eram respectivamente: acesso a materiais de treinamento, comunicação via MSN ou Google Talk, acesso a Facebook, Twitter e a informações de sites como Wikipédia e RSS. A interface para entrada e saída de informação no aplicativo, descrita no artigo, executava as seguintes ações: tradução de um símbolo para o alfabeto Braille, tradução de símbolo Braille para vibração ao receber mensagem ou gesto de um usuário. A interface usada para controlar o dispositivo executava a seguintes funções: ajuste de velocidade de leitura, retorno da velocidade de leitura e definição de alguns parâmetros do aplicativo. Os autores afirmaram que o projeto de software foi construído de forma integrada e ao final propuseram melhorias a serem desenvolvidas: suporte para câmeras web para reconhecimento de gestos como entrada de dados para o usuário; sincronização entre PC e versão móvel; desenvolvimento de um módulo para jogos; desenvolvimento do aplicativo móvel para plataforma Android e Iphone.

Outro artigo interessante, o de Hossny et al. (Hossny et al., 2015) propõe um framework para comunicar a performance artística de bailarinos para pessoas surdas, cegas e surdocegas que estejam na plateia. A apresentação para as pessoas com 
deficiência acontece na forma háptica através do sentido do toque como ilustrado na figura 3. O framework transmitia o movimento da dança subdividido em três módulos principais: a captura de movimento, a tradução do movimento para a interação háptica e a exibição num dispositivo. A estrutura proposta analisava o movimento da dança, transmitia a localização espacial e reconhecia os passos da dança que eram comunicados através de uma grade de vibradores que eram transmitidos de forma háptica usando um robô humanoide. A captura dos movimentos da dança era feita através de câmeras de captura de luz infravermelha. $\mathrm{O}$ artista intérprete ou executante possuía marcadores reflexivos de luz infravermelha em diferentes partes do corpo, as câmeras capturavam as trajetórias dos marcadores. Além das câmeras, usou-se também um Sistema de Posicionamento Global Interno. O módulo de tradução consistia na segmentação do movimento: análise, reconhecimento e mapeamento para uma resposta háptica. Ao obter a resposta háptica, esta era renderizada para o dispositivo háptico (robô) e transmitida ao indivíduo com deficiência.

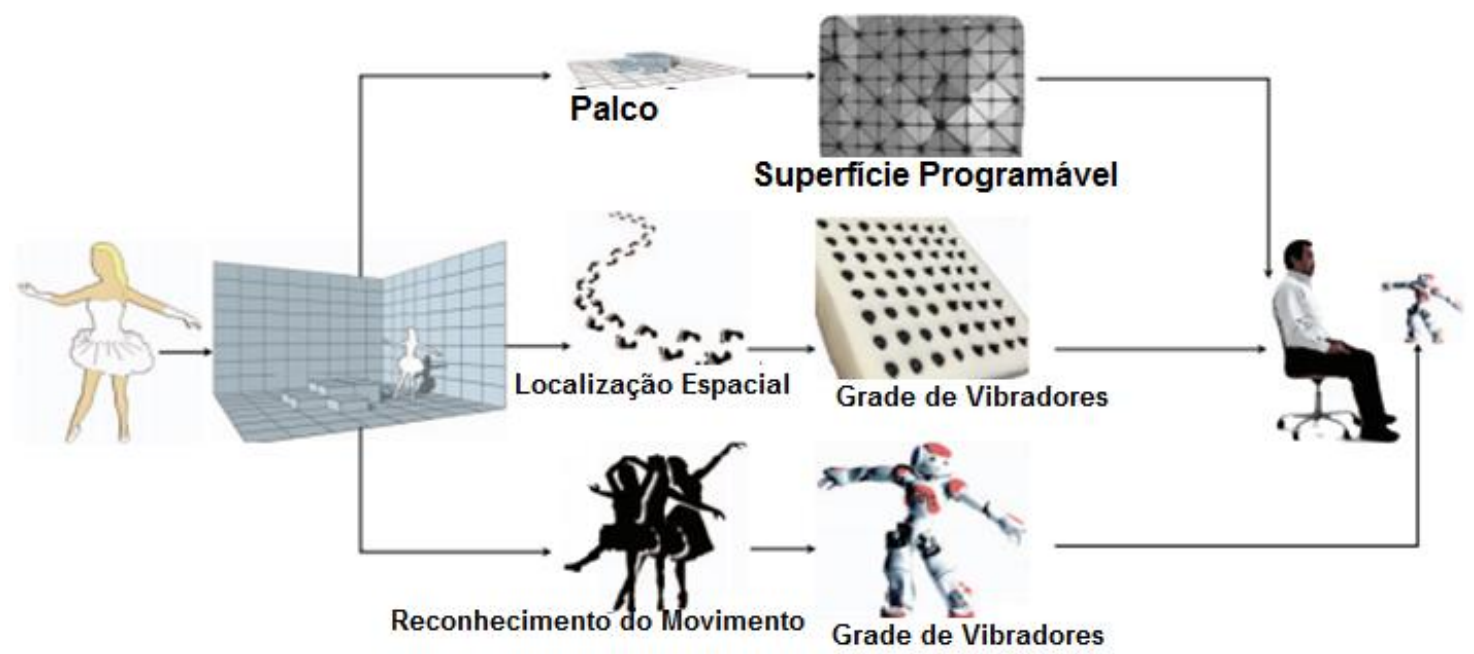

Figura 3. Esquema do Framework e Robô com dispositivo háptico.

Fonte: Hossny, et al., 2015.

Analisando os países de publicação (figura 2), o Japão liderou o ranking de publicações encontradas na busca na base Scopus. Dos dezenove artigos selecionados do Japão, nove artigos foram publicados pelo mesmo grupo de pesquisadores (Ohtsuka, et al.), de 2008 a 2015, (Ohtsuka et al., 2015;Ohtsuka et al., 2014; Ohtsuka et al., 2012; Ohtsuka et al., 2010; Ohtsuka et al., 2008). Assim pode-se perceber a evolução gradual da pesquisa. Em 2008, Ohtsuka et al. (Ohtsuka et al., 2008A e 2008B) publicou um artigo fruto de pesquisas desde 2002, apresentando o dispositivo B-brll dispositivo de bodybraile, baseado num sistema motor de duas vibrações e um telefone celular para transmitir os códigos de bodybraile. Vários experimentos foram realizados. Um deles combinou o sistema de telefone de TV de um celular G3 e o dispositivo B-brll. Se uma pessoa surdocega que vive sozinha desejasse realizar uma tarefa com necessidade de utilização de meios visuais, como por exemplo, ler um e-mail ou confirmar a quantidade de líquido contida numa garrafa, ela poderia obter essas informações através do envio de um filme para uma equipe de suporte de visão remota através de um sinal DualTone-Multiple-Frequency (DTMF) e receberia dados traduzidos para o bodybraile de volta. Os resultados obtidos foram confiáveis através dos experimentos. 
Em 2010, o grupo apresentou à comunidade um sistema de comunicação bidirecional baseado num colete com sensores infravermelhos e um dispositivo portátil semelhante ao teminal de digitar senhas de caixas de banco, Ohtsuka et al. (Ohtsuka et al., 2010A e 2010B). Em 2012 eles apresentaram o "telefone Helen Keller", ou P-Bbrll dispositivo de bodybraile para bolso, um sistema de comunicação via Skype, Ohtsuka et al. (Ohtsuka et al., 2012A e 2012B). Em 2014, apresentaram experimentos para a transmissão de notas musicais com a utilização do dispositivo P-Bbrll, Ohtsuka et al.. (Ohtsuka et al., 2014A) e também divulgaram o uso de Liga de Memória de Forma ao invés de microvibradores no dispositivo, que permitiu flexibilizar a modulação por largura de pulso (MLP), Ohtsuka et al. (Ohtsuka et al., 2014B). Em 2015 publicaram o resultado de uma análise da acurácia do dispositivo com relação à frequência de vibração e amplitude, o que possibilitou sugerir qual seria a melhor banda de frequência para o dispositivo, Ohtsuka et al. (Ohtsuka et al., 2015).

O trabalho de Ohtsuka et al. (Ohtsuka et al., 2008A e 2008B) poderia ser utilizado com um aplicativo similar ao aplicativo gratuito Be My Eyes (Wiberg, 2015), criado pelo dinamarquês Hans Jorgen Wiberg, onde voluntários podem emprestar sua visão para quem tem alguma deficiência visual e precisa de ajuda quando está sozinho em situações do dia a dia, tais como, ler o vencimento de uma fatura, validade de medicamentos ou produtos, e até mesmo a opinião numa combinação de cores e roupas. Porém o aplicativo Be My Eyes, funciona com voz e vídeo ao vivo, assim, voluntários e deficientes visuais precisam estar conectados à internet. Uma proposta de estudo futuro seria uma ampliação do aplicativo para Be My Ears and Eyes, para atender aos surdocegos.

Ainda utilizando como base a ideia de um celular que contenha um aplicativo para tradução bodybraile, se o incrementasse com inteligência artificial, se teria um produto similar ao Aipoly Vision (Aipoly, 2016), desenvolvido pela empresa Aipoly, especializada em inteligência artificial, disponível nas lojas de aplicativos para celulares iOS e Android. O Aipoly Vision reconhece o objeto, cena ou a cor para a qual a câmera do celular é apontada, e identifica a imagem obtida em áudio para o usuário. Portanto, se a informação produzida fosse traduzida para um dispositivo vibrotátil, poderia obter uma nova versão do aplicativo, o Aipoly Vision\&Hearing. E tais aplicativos poderiam ser utilizados e testados para fins pedagógicos.

Esses artigos não estavam diretamente relacionados ao uso de tecnologias para a comunicação e interação de surdocegos no processo ensino-aprendizagem, bem como os demais artigos analisados. Mas essas iniciativas poderiam transmitir de alguma forma conhecimento para os surdocegos, seja por aprendizado de notas musicais ou por passos de danças. Eles evidenciam importantes propostas com diferentes enfoques para a realização da comunicação com surdocegos entre si ou com pessoas sem deficiência, mas que poderão servir de ideia para um a futura investigação no campo de tecnologias aplicadas à comunicação de surdocegos num processo de ensino-aprendizagem.

A comunicação foi o tema mais presente nas pesquisas dos artigos avaliados e isto se deve ao fato deste ser o principal obstáculo à pessoa surdocega. Enquanto ela não adquire uma forma de comunicação, fica num isolamento e não desenvolve a sua capacidade cognitiva. Quanto mais dispositivos existirem para que ela se comunique, reduzindo os fatores que condicionam suas limitações, mais fácil poderá ser a sua trajetória educacional. 


\section{Tecnologias e os Métodos Identificados para Comunicação de Pessoas Surdocegas}

Ao término do levantamento bibliográfico foi possível identificar as tecnologias abordadas bem como os métodos que foram sistematizados como a língua e os dispositivos adotados nos estudos (Tabela 3 ).

\section{Tabela 3. Classificação dos artigos quanto as tecnologias Fonte: Elaborado pelos autores}

\begin{tabular}{|l|c|}
\hline Tecnologias & N $^{\mathbf{0}}$ de Artigos \\
\hline Vibrotátil & 38 \\
\hline Multimídias & 14 \\
\hline WEB & 9 \\
\hline Tátil & 9 \\
\hline Arduíno & 5 \\
\hline Bluetooth & 5 \\
\hline GPS & 3 \\
\hline RFID & 1 \\
\hline
\end{tabular}

$\mathrm{Na}$ categoria Vibrotátil foram considerados dispositivos táteis e vibrotáteis. Em Multimídias foram considerados os recursos de vídeo (câmeras), áudio (microfones, sintetizadores de voz), controle de videogame e piso tátil. Em WEB foram considerados dispositivos que funcionavam online como, por exemplo, os experimentos com comunicação em tempo real utilizando o software skype ou outro tipo de programas para bate-papo. Em Tátil, dispositivos somente táteis. Em Arduíno, dispositivos que usavam hardware arduíno. Nas categorias Bluetooth e GPS foram considerados dispositivos vibrotáteis que utilizaram essa forma de transmissão de dados. Em RFID foi considerado uma pesquisa que estava relacionada à dispositivo vibrotátil, que recebia dados remotamente através de etiquetas de identificação por radiofrequência.

Os dispositivos desenvolvidos ou utilizados nos estudos foram categorizados em Smartphones quando utilizavam celulares com tela de toque. Dispositivos vestíveis, aqueles que poderiam ser colocados em outras partes do corpo diferente do punho, cuja classificação foi considerada Smartwatch. Robôs os dispositivos desenvolvidos como mão robótica, robô humanoide ou robô de quatro ou três pés. Na categoria "outros" foram classificados todos os dispositivos que foram desenvolvidos ou utilizados na pesquisa diferente dos anteriores. Dentre eles identificou-se tablets, dispositivos com ou sem células piezoelétricas e dispositivos com controle de videogame (Tabela 4).

Tabela 4. Classificação dos artigos quanto aos dispositivos. Fonte: Elaborado pelos autores

\begin{tabular}{|l|c|}
\hline \multicolumn{1}{|c|}{ Dispositivos } & $\mathbf{N}^{\mathbf{0}}$ de Artigos \\
\hline Smartphones & 15 \\
\hline Vestível & 11 \\
\hline Smartwatches & 2 \\
\hline Robôs & 5 \\
\hline Outros & 18 \\
\hline
\end{tabular}

Para categorizar as línguas ou códigos utilizados nos trabalhos considerados, foram incluídos na categoria Braile, o sistema Braile e suas derivações, bodybraile, 
fingerbraile. Na categoria Sinais, a Língua de Sinais do país de origem da pesquisa, ou outro tipo, como por exemplo, a Kana, sistema japonês de escrita silábica. Em Morse, trabalhos que se basearam no sistema de representação do Código Morse. Alfabeto manual, pesquisas que utilizaram a soletração nos dedos e palmas das mãos como o Sistema Malossi e o Lorm. Na categoria outras, foram incluídas codificações diferentes das classificações anteriores, pois exploravam os sentidos somáticos como a percepção do movimento de uma dança ou a atividade de cavalgar. Dos 49 artigos analisados não foram identificadas língua ou utilização de códigos em três artigos (Tabela 5).

Tabela 5. Classificação dos artigos quanto à língua/código. Fonte: Elaborado pelos autores

\begin{tabular}{|l|l|}
\hline Língua / Código & $\mathbf{N}^{\mathbf{0}}$ de Artigos \\
\hline Braile & 28 \\
\hline Sinais & 9 \\
\hline Morse & 3 \\
\hline Alfabeto Manual & 3 \\
\hline Outras & 3 \\
\hline
\end{tabular}

\section{Conclusão}

A presente pesquisa permitiu conhecer questões básicas sobre a surdocegueira e algumas produções científicas internacionais na área de Sistemas de Computação que abordem esse tema. Identificou que diversas pesquisas estudam e desenvolvem ferramentas tecnológicas que explorem formas de interação e métodos para potencializar a comunicação de indivíduos surdocegos com outras pessoas. Por ser tratar de uma comunicação onde os usuários apresentam necessidades multissensoriais, muitos desses trabalhos tem características multimodais abordando assim diferentes técnicas de interação em conjunto.

A partir deste trabalho foi possível identificar as tecnologias, as linguagens e os tipos de dispositivos (artefatos de TI) citados nos artigos e encontrados na revisão bibliográfica que são utilizados na comunicação dos surdocegos entre si ou com pessoas sem deficiência e que poderiam auxiliar na educação dos indivíduos surdocegos.

$\mathrm{Na}$ história da educação dos surdocegos, a comunicação é crítica no desenvolvimento deste indivíduo (Amaral, 2002). Sendo assim entende-se que estudos de novas tecnologias baseadas em eletrônica e computação adaptadas aos celulares, podem ser novas formas de promover a comunicação sensorial efetuada através do tato.

É possível perceber a tendência atual de uso de dispositivos móveis utilizando prioritariamente smartphones, smartwatches e tecnologias vestíveis com o uso de recursos vibrotáteis e outros inerentes dessas tecnologias como recursos multimídias de câmera, microfone e geolocalização como o GPS.

Os trabalhos que abordam outros tipos de dispositivos exploraram a criação de seus próprios hardware/software como foi o caso de alguns trabalhos que foram desenvolvidos com arduíno e objetos robóticos.

É interessante observar que embora a maioria dos trabalhos estivesse relacionada a tecnologias que tratavam da comunicação bidirecional, muitos deles não exploraram a aprendizagem. Desta forma, não é possível afirmar que efetivamente melhorem o desenvolvimento do surdocego num processo de ensino-aprendizagem. Esta pesquisa 
permitiu constatar que há ainda muitas possibilidades de pesquisas na área de utilização de meios alternativos de comunicação associados a recursos pedagógicos, visando o desenvolvimento de conteúdos programáticos de áreas acadêmicas para o surdocego.

O maior volume de publicações foi feito pelos seguintes países desenvolvidos: Japão, Itália e Estados Unidos. Também foi observado que a Índia, integrante do BRICS (Brasil, Rússia, China e África do Sul), grupo de países em desenvolvimento, teve o mesmo número de artigos publicados em eventos internacionais que o Reino Unido, país desenvolvido.

A pesquisa foi realizada com palavras-chaves no idioma em inglês, somente na base de dados Scopus, numa primeira parte da revisão, cuja busca de dados foi restrita a títulos de estudos internacionais e num segundo momento, será realizada uma revisão de abrangência no Brasil.

De acordo com a pesquisa bibliográfica realizada por (Bertola, 2014), a comunicação é o tema mais abordado nas dissertações e teses de programas nacionais que pesquisaram sobre a surdocegueira. Este trabalho analisou as produções científicas apresentadas à comunidade científica brasileira no período de 2003 a 2013. Fazendo uma comparação com o que foi encontrado neste primeiro levantamento bibliográfico com trabalhos internacionais, a comunicação é sempre o ponto base para $o$ desenvolvimento das pesquisas.

Este estudo permitiu que conhecêssemos questões básicas sobre a surdocegueira e as produções científicas internacionais na área de Sistemas de Computação apoiando a Surdocegueira. Como intenção de trabalhos futuros, estão as seguintes ações:

- Realizar a pesquisa na mesma base de dados desta primeira revisão, Scopus, com palavras-chaves em português;

- Realizar a pesquisa com termos em português na base de dados de dissertações e teses da Capes, utilizando as áreas de conhecimento Educação combinada com Ciências da Computação, restrita a estudos brasileiros, publicados tanto em português quanto inglês.

Assim, busca-se saber qual o estado da arte das pesquisas desenvolvidas em tecnologias voltadas para a surdocegueira. E também identificar como é feita a comunicação entre surdocegos e/ou pessoas sem deficiência num processo ensino-aprendizagem, a partir de estudos que relacionem as duas áreas: Educação e Ciência da Computação, de forma a buscar subsídios para identificar métodos de ensino com tecnologias para surdocegos.

\section{Referências}

Aipoly Vision. Versão 1.0. Estados Unidos: Aipoly Inc., 2016. Disponível em: $<$ http://aipoly.com/>. Acesso em: 07 de maio de 2017.

Aitken, S. Understanding deafblindness. In: Teaching children who are deafblind. Contact, communication and learning. London: David Fulton Publishers Ltd., p. 01$34,2000$.

Amaral, I. A Educação de Estudantes com Surdocegueira. In: MASINI, E.F.S. Do sentido, pelos sentidos para os sentidos. Niterói, Intertexto, p.124, 2002. 
Araujo, R., Suzana, R., Boscarioli, C. I GranDSI-BR - Grandes Desafios de Pesquisa em Sistemas de Informação no Brasil 2016 a 2026. 2017. Disponível em:< https://www.researchgate.net/publication/314856566_I_GranDSI-BR_-_Grandes_De safios_de_Pesquisa_em_Sistemas_de_Informacao_no_Brasil_2016_a_2026 >. Acesso em: 26 de março de 2018.

Arduino. In: Wikipédia: a enciclopédia livre. Disponível em: $<$ https://pt/m/wikipedia .org/wiki/Arduino> . Acesso em: 19 de março 2018.

Bertola, V.; Costa, M. P. R. Surdocegueira congênita: análise sobre pesquisas e publicações no Brasil (período 2003-2013). In: VI Congresso Brasileiro de Educação Especial - VI CBEE, IX Encontro Nacional dos Pesquisadores da Educação Especial - IX ENPEE, 2014. Anais do VI Congresso Brasileiro de Educação Especial, 2014.

Blumer, H. A Natureza do Interacionismo Simbólico. In: MORTENSEN, C.D. Teoria da comunicação: textos básicos. São Paulo: Mosaico, p. 119-138, 1980.

Cader-Nascimento. F.A.A.A, Costa M.P.R., Descobrindo a Surdocegueira: Educação e Comunicação. São Carlos. EdUFSCar, 2010.

Ferreira, A. J. Avanços das Políticas Públicas para as Pessoas com Deficiência. Uma análise a partir das Conferências Nacionais. $1^{\circ}$ edição. BRASÍLIA, 2012. Disponível em: $<$ http://www.pessoacomdeficiencia.gov.br/app/sites/default/files/publicacoes/livr o-avancos-politicas-publicas-pcd.pdf $>$. Acesso em: 19 de maio de 2017.

Ferreira, S.B.L.; Saramento, C; Alves, A.S., Leitão, C.S., Maciel, D.R., Matos, S.N. e Britto,T.C.P.. Accessibility and Digital Inclusion: Utopia or a Great Challenge? In Proceedings of the 16th Brazilian Symposium on Human Factors in Computing Systems (IHC'17). Brazilian Computer Society, Joinville, Santa Catarina, 2017. Disponível em: $<$ https://www.researchgate.net/publication/320715904_Accessibility_and_Digital_In clusion_Utopia_or_a_Great_Challenge $>$. Acesso em: $\overline{1} 9$ de março de $201 \overline{8}$.

Frederico, C. E. O domínio de atividades da vida autônoma e social referentes à alimentação de crianças surdocegas com fissura lábio palatal. Dissertação de Mestrado. Universidade Presbiteriana Mackenzie. Curso de Pós Graduação de Distúrbios do Desenvolvimento. São Paulo, 2006.

Garcia, A. Surdocegueira: Empírica e Científica, 2008. Disponível em: $<\mathrm{http}$ //www.agapasm.com.br/surdocegueiraempiricaecientifica/obra.asp $>$. Acesso em: 13 de outubro de 2016.

Gorodnitsky, D., Kalashnikoff, V., Pavliy, E., Pinchuk, E., Tumanoff, A., Tumanova, O., Ustyan, N. Hardware-software complex "dashpoint" for learning and communication of deafblind people. ICETA 2011 - 9th IEEE International Conference on Emerging eLearning Technologies and Applications, Proceedings, p. 95-98, 2011.

Granatto, C.; Pallaro, M; Bim, S. Digital Accessibility: Systematic Review of Papers from the Brazilian Symposium on Human Factors in Computer Systems. In Proceedings of the 15th Brazilian Symposium on Human Factors in Computing Systems (IHC'16). Brazilian Computer Society, São Paulo, Brazil, 2016. Disponível em: $<$ http://dl.acm.org/citation.cfm?id=3033722>. Acesso em: 19 de maio de 2017. 
Grupo Brasil De Apoio Ao Surdocego E Ao Múltiplo Deficiente Sensorial Informe Nacional Sobre a Exclusão das Pessoas Surdacegas no Brasil. Tradução: Lilia Giacomini. São Paulo, Abril, 2003.

Hossny, M., Nahavandi, S., Fielding, M., Mullins, J., Mohamed, S., Creighton, D., Mccormick, J., Vincs, K., Vincent, J., Hutchison, S. Haptically-enabled dance visualisation framework for deafblind-folded audience and artists. Proceedings 2015 IEEE International Conference on Systems, Man, and Cybernetics, SMC 2015, p. 446-450, 2015.

Mcinnes, JM. Deafblindeness: a unique disability. In: A guide to planning and support for individuals who are deafblind. Canada: University of Toronto Press Incorporated, p. 03-33, 1999.

Nascimento, F.A.A.A.C.. Educação infantil ; saberes e práticas da inclusão : dificuldades de comunicação e sinalização : surdocegueira/múltipla deficiência sensorial.4 ${ }^{\mathrm{a}}$ edição. E Shirley Rodrigues Maia - Associação Educacional para a Múltipla Deficiência - AHIMSA. - Brasília : MEC, Secretaria de Educação Especial, 2006. Disponível em: http://portal.mec.gov.br/seesp/arquivos/pdf/surdosegueira.pdf. Acesso em: 13 de outubro de 2016.

Ohtsuka, S.A, Sasaki, N.B, Hasegawa, S.C, Harakawa, T.D. Body-braille system for disabled people. Lecture Notes in Computer Science (including subseries Lecture Notes in Artificial Intelligence and Lecture Notes in Bioinformatics), 5105 LNCS, p. 682-685. 2008A.

Ohtsuka, S.A, Sssaki, N.B, Hasegawa, S.C, Harakawa, T.D. The introduction of telesupport system for deaf-blind people using body-Braille and a mobile phone. 5th IEEE Consumer Communications and Networking Conference, CCNC 2008, p. 1263-1264. 2008B.

Ohtsuka, S.A, Sssaki, N.B, Hasegawa, S.C, Harakawa, T.D. Development of a musical score system using Body-Braille. Lecture Notes in Computer Science (including subseries Lecture Notes in Artificial Intelligence and Lecture Notes in Bioinformatics), 6180 LNCS (PART 2), p. 443-446. 2010A.

Ohtsuka, S.A, Sssaki, N.B, Hasegawa, S.C, Harakawa, T.D. Communication system between deaf-blind people and non-disabled people using Body-braille and infrared communication. 7th IEEE Consumer Communications and Networking Conference, CCNC 2010. 2010B.

Ohtsuka, S.A, Sssaki, N.B, Hasegawa, S.C, Harakawa, T.D. Introduction of new bodyBraille devices and applications. Lecture Notes in Computer Science (including subseries Lecture Notes in Artificial Intelligence and Lecture Notes in Bioinformatics), 7383 LNCS (PART 2), p. 672-675. 2012A.

Ohtsuka, S.A, Sssaki, N.B, Hasegawa, S.C, Harakawa, T.D. Helen Keller Phone - A communication system for deaf-blind people using Body-Braille and Skype, 2012 IEEE Consumer Communications and Networking Conference, CCNC'2012, , p. 3031. 2012B.

Sasaki, N.A, Ohtsuka, S.B, Ishii, K.C, Harakawa, T.D. The development of a music presentation system by two vibrators. Lecture Notes in Computer Science (including 
subseries Lecture Notes in Artificial Intelligence and Lecture Notes in Bioinformatics), 8547 LNCS (PART 1), p. 602-605. 2014A.

Sasaki, N.A, Nakajima, K.A, Ohtsuka, S.B, ISHII, K.C, HARAKAWA, T.D The study of a new actuator for a two-point Body-Braille system. Lecture Notes in Computer Science (including subseries Lecture Notes in Artificial Intelligence and Lecture Notes in Bioinformatics), 8547 LNCS (PART 1), p. 630-633. 2014B.

Soares, R. A História da Educação do Surdocego no Brasil. In: Toque: Mãos Que Falam. Ano 1, No 1, São Paulo, 1999.

Sherman, W e Craig, A., Understanding Virtual Reality. Morgan Kaufmann Publishers, San Francisco, CA, p. 266, 2003.

Vieira, A.L.N., Novaes, F.F., Silva, D.M., Santos, L., Belozi, S., Castro, T. A mobile solution for linguistic communication with deaf-blind people using Arduino and Android. Proceedings of the 11th International Network Conference, INC 2016, p. 177-180, 2016.

Vosgerau, D. S. R.; Romanowski, J. P. Estudos de revisão: implicações conceituais e metodológicas. Rev. Diálogo Educ., Curitiba, v. 14, n. 41, p. 165-189, jan./abr., 2014. Disponível em:< http://www2.pucpr.br/reol/index.php/dialogo?dd99=pdf\&dd1 $=12623>$. Acesso em: 07 de maio de 2017 .

Wiberg, H. J.. Be My Eyes. Dinamarca: BE MY EYES, 2015. Disponível em: $<\mathrm{http}: / /$ bemyeyes.com/>. Acesso em: 07 de maio de 2017.

Zeza, M.A, Stavrou, P. D. Program of educational intervention for deaf-blind students. Lecture Notes in Computer Science (including subseries Lecture Notes in Artificial Intelligence and Lecture Notes in Bioinformatics), 9142 LNCS, p. 472-478, 2015. 